\title{
La notion de période énonciative: L'exemple des expressions verbales
}

\author{
María Luisa Fernández Echevarría \\ Universidad Complutense de Madrid / Modyco \\ luisafernandez@ucm.es / fernandez.1f09@gmail.com
}

\begin{abstract}
Résumé. La période énonciative sert à expliciter certains caractères formels des segments idiomatiques répertoriés dans un grand nombre de langues. Ils ne sont pas considérés des unités lingüistiques car leur caractérisation échappe à un modèle syntaxique unique dans le métalangage de la grammaire. D'autre part, le statut d'unités lexicales (mots) ne leur convient pas non plus car ils signifient autrement que par le contexte extralinguistique immédiat et s'organisent en chaînes verbales à longueur variable, à l'instar des syntagmes. Après avoir défini quelques-uns de ces segments (les parémies) par leur typologie syllabique (assonances, récurrences), nous entreprenons dans ce travail une étude d'instances qui dépendent d'un élément caractérisé par sa morphologie : le verbe. En nous aidant d'un corpus complémentaire de mots d'esprit, l'étude contrastive de quelques phraséologies verbales, donne pour résultat surprenant la disparité des formes correspondant aux traductions. Surprenant aussi le fait que des notions poussées sur l'aspect verbal, en rapport avec celle d'ouverture/fermeture énonciative, permettent d'associer à leurs contextes un espace syntaxique/prosodique dans le locus discursif de leur utilisation. Nous concluons que les rapports sémantiques des éléments qui les composent, ont un caractère domanial et médiatisé servant à redéfinir la notion de métaphore en parémiologie. Cette étude nous semble pertinente en traductologie et acquisition des langues par les classifications syntaxiques qu'elle suggère en cohérence avec les critères présents dans les grammaires de certaines langues.
\end{abstract}

\begin{abstract}
The term utterance period is used to explain certain formal characteristics of idiomatic clusters, as listed in a variety of languages. They can not be considered as linguistic units, because their characterizations escape unique syntactical modelling in grammar meta-language. Hence, they don't have the status of tokens (words) in the sense that they mean otherwise that by the immediate extra-linguistic context and are organized on variable length verbal chains as syntagmatic structures. After defining some of these segments (paremies) by their syllabic type (assonances, recurrences), we undertake in this work the study of instances which typology is depending in one morphological element: the verb. Also based on a complementary corpus of quips, this contrastive study of some phrasals verbs gives the surprising result of the disparity shapes corresponding to translations. It also surprises that an extensive notion of verbal aspect, connected to opening/closing utterance patterns, indicate syntactic/prosodic positions in their contexts that operate as discursive locus in actual speech. We conclude that semantic relationships of elements in idioms have a meaning because of their mediatized domanial field (domaine) which allows the redefinition of metaphor in paremiology. The study seems finally relevant in translation and language acquisition because it suggests new syntactic classifications in line with grammatical categorisations of some languages.
\end{abstract}

\section{Introduction}

Cet article se compose de 4 parties. Dans la première nous attribuons un caractère domanial à certaines chaînes segmentales répertoriées dans les langues comme des clusters sémantiques ininterprétables directement par la référence prototypique (Kleiber: 1999) d'un de ses éléments nucléaires. Cette caractérisation domaniale représente une conclusion à séquence discursive qui les rend autonomes du cotexte où elles sont insérées. 
Nous présentons l'extrait d'un corpus de 82 jeux de mots et les analysons tant par leur caractère syllabique (récurrences) que par leurs reprises anaphoriques. Une reprise dé-contextualisée (énonciation anaphorique) les rend inusuelles, et donc signifiantes, par la réprésentation iconique d'un élément dans la deuxième partie dont la formulation est déviante par rapport au statut grammatical accordé à la première partie. Nous caractérisons trois types de procédures sémiologiques qui justifient les doubles sens par un mouvement linéaire impliquant des récurrences syllabiques et des dédoublements à caractère métaphorique.

Dans la deuxième partie nous retenons les conclusions faites sur un corpus de parémies et formulons des catégories morphologiques qui sous-tendent des représentations iconiques par la confrontation d'intances temporelles. Il s'agit de lexies dont les flexions d'un des éléments s'oppose dans les deux parties de ces expressions caractérisés par la binarité. Cette deuxième analyse prépare nos conclusions sur les paraphrases verbales. Nous découvrons en effet que les rapports établis par des éléments nucléaires à morphologie (verbes) peuvent se définir selon une combinatoire syntaxique qui rappelle celle d'une logique de premier ordre (implication $\Rightarrow$; implication inverse $\Leftarrow$; double implication $\Leftrightarrow$ et exclusion $/ \Rightarrow$ ) similaire à celle que Martin (1979) décrit pour parler des rapports intonatifs entre des clusters syllabiques signifiants. Nous rapprochons alors cette combinatoire basée sur les instances temporelles de la notion de verbe support (Seong Heon Lee: 2011) et considérons l'aspect verbal comme un opérateur de structures intonatives nucléaires justifiant une méso-syntaxe d'où émergeraient des constantes sémiologiques. C'est dans ce cadre que des segments bornés ou faits linguistiques trouvent leur place pour représenter des unités linguistiques (figements) que nous appelons périodes énonciatives. Les jeux de mots, parémies et phraséologies verbales, sont des exemples de ces unités linguistiques qui s'opposent aux faits de langue par leur relative instabilité et leur définition floue en termes d'une grammaire de langue. Dans la troisième partie, nous présentons finalement des phraséologies verbales autour des verbes llevar [porter], dar [donner], tomar [prendre], coger [saisir], poner [mettre], soler [avoir l'habitude de], et annonçons ce qui nous semble le résultat crucial de notre analyse: leur intraductibilité sur un même support verbal mais répondant aux mêmes conditions téliques (aspect résultatif ouvert ou fermé) dans le locus de la période qui les inclut. Nous concluons que leur sens et donc déterminé par une référence domaniale horizontale (récurrence à des situations extra-linguistiques) et non pas à un axe vertical de signifiés (paradigmatique). Les variables phonologiques concernant l'articulation intonative en termes d'accent initial/accent final $(\mathrm{AI} / \mathrm{AF})$ servent alors à élaborer des contextes cadre dans le domaine de la traduction automatique, ou de la médiation linguistique en général, car les périodes énonciatives sont des faits linguistiques qui délimitent l'ordre des images acoustiques par les présentations iconiques qu'elle produit au moment de l'énonciation. La structure superficielle d'une langue devient donc un ensemble de faits formels modélisés dans le langage d'une grammaire catégorielle dynamique et falsable.

\section{Catégories super-ordonnées et jeux de décontextualisation}

Nous impruntons à Kleiber (2015) la notion de catégorie d'idiomes super-ordonnés; elle permet de référer à une situation extra-linguistique précise et de rendre explicite leur interprétation sémantique. Nous proposons d'aborder avec elle les expressions idiomatiques qui ne peuvent pas être analysées par leur caractère strictement syllabique (rimes, assonances, récurrences lexicales...). Dans le même temps nous établissons de critères pour l'analyse des paraphrases verbales qui ne font pas partie des répertoires des sentences propres à la parémiologie. Cette catégorie émergente par défaut ${ }^{1}$, se base sur l'élément constitutif flexif (le verbe dans le travail qui nous occupe) qui doit être instancié dans chaque production. S'intégrant dans le discours naturellement, les phraséologies verbales que nous présentons conforment des segments linguistiques au delà du mot, mais ne constituent pas à proprement parler des syntagmes car ils n'arrivent pas à se constituer en termes nucléaires comme les mots lexicaux et sont idéntifiés seulement comme des lexies dans le sens de Legallois (2012). L'approche par la métaphore, étant très poussée pour les idiomes, nous entreprenons l'analyse interne d'un corpus de jeux de mots, en utilisant les outils de l'analyse prototypique, pour dégager les critères de colligation des phraséologies verbales. Le résultat sert de piste à la conception de paradigmes formels pour établir des répértoires sur la base de représentations iconiques qui émergent à l'intérieur d'instances syllabiques bornées témoignant des intuitions sémiologiques partagés par les locuteurs d'une langue. Il ne faut pas recourir à une supposée 
compétence virtuelle de la grammaire pour justifier le fait que le locuteur natif est capable de repérer des collocations qu'il n'a jamais utilisées ou entendues, il s'agit de la même capacité phonologique qui le rend capable de distinguer entre énoncés a-significatifs d'un côté et déviants de l'autre, para rapport à un usage normatif consolidé mais soumis à la variation. En effet, dans un travail sur les énoncés asignificatifs de la traduction automatique (Fernández 2015b), il a été montré que ce qui provoque la dislocation dans la chaîne verbalisée, peut être analysé par les déviances strictement syllabiques, ce qui n'est pas le cas des énoncés déviants par rapport à l'usage d'un locuteur natif dont les locus ${ }^{2}$ prosodiques répondent à des positions syntaxiques probables. Nous avons retenu cet argument concernant la morphophonologie et que supportent les constantes phono-tactiques de la constituance syllabique, ainsi que celles qui s'établissent entre les mots (enchaînements, ou même liaisons dans le cas du français ${ }^{3}$ ) pour mettre en évidence les clusters qui constituent les phraséologies. Si les ruptures syntaxiques provoquant des nonsens dans la traduction automatique rend compte de ce qu'on pourrait appeler dislexie métaphorique dans la linéarité discursive, ces mêmes ruptures gérés dans des contextes syllabiques cohérents rendent compte, comme nous allons le voir des colligations nécessaires à expliquer la composante linguistique des jeux d'esprit et d'autres (dé)-figements. Considérons alors quelques mots d'esprits de notre corpus complémentaire que nous répertorions dans les 5 catégories du tableau 1; le cadre sémiologique qui les rend possibles en tant que jeux de mots significatifs opèrent par l'intéraction de contextes déviants associés. Cela est possible grâce au cadre sémiologique commun qu'une machine virtuelle ne peut pas encore reconstruire mais auquel le locuteur humain ne peut échapper.Voyons des exemples ${ }^{4}$ de métastase métaphorique 5 .

\subsection{La représentation iconique de la syllabe dans les jeux de mots à caractère phonologique.}

Pour établir des catégories dans les jeux de mots, nous nous sommes basés sur des critères qui tiennent compte du caractère linéaire du signal linguistique et de la mémoire phonologique. Tous les exemples montrent que les deux instances, question et réponse, sont médiatisées dans le temps et rapprochés par des critères autres qu'un cadre contextuel commun. L'instanciation implique le rapprochement des deux qui est proposé par le locuteur et doit être confirmé par l'allocutaire. (1) à (9) exigent la reconnaissance d'instances lexicales plus ou moins étendues, parfois culturelles $(1,4,5)$ alors que $(3,4,6,7,8)$ sont interprétables seulement par la connaissance du code orthographique de la langue. Dans le cas de formules à déplacement syllabique elles présentent aussi une déformation du code qui doit être acceptée par l'alocutaire.

Tableau 1

Rapports phonologiques

\begin{tabular}{|c|c|}
\hline MODALITÉ & EXEMPLE \\
\hline Mouvement linéaire (Extension) & $\begin{array}{l}\text { 1. Pourquoi le foot c'est rigolo? } \\
\text { Parce-que Thierry en rit! } \\
\text { 2. Quel est le fruit le plus féminin? } \\
\text { L'ananas! } \\
\text { 3. Que dit un oignon quand il se cogne? } \\
\text { Aïe! } \\
\text { 4. Quel est le sport préféré des insectes? } \\
\text { Le criquet! } \\
\text { 5. Pourquoi Michaël ouvre la porte? } \\
\text { Parce que Jack sonne. (Jackson) } \\
\text { 6. Qui du marin ou de l'aviateur écrit le moins? } \\
\text { Réponse: le marin car il a jeté l'ancre! } \\
\text { 7. Quelles sont les lettres qui bougent tout le temps? } \\
\text { AJT (agiter) } \\
\text { 8. Quelles sont les lettres qui se voient le moins? } \\
\text { FAC (effacer) }\end{array}$ \\
\hline Déplacement syllabique (geste) & 9. Que font deux brosses à dents le 14 juillet? \\
\hline
\end{tabular}




\begin{tabular}{|c|c|}
\hline & $\begin{array}{l}\text { Un feu dentifrice! } \\
\text { 10. *Un petit nuage se promène avec sa maman dans le ciel. } \\
\text { Tout à coup, il s'arrête en se tortillant: } \\
\text {-J'ai envie de faire pluie-pluie, maman! } \\
\text { 11. Quelle est l'étoile la plus sale? } \\
\text { La toile d'araignée } \\
\text { 12. Oui M.le commissaire, mon père et maire et ma tante soeur } \\
\text { J'ai un cousin qui est frère et mon frère est masseur } \\
\text { 13. La maîtresse demande de construire une phrase avec } \\
\quad \text { l'adjectif épithète. } \\
\text { Nicolas lève le doigt et dit : } \\
\text { Aujourd'hui il pleut, épithète (et peut-être) demain, il fera beau! } \\
\text { 14. Quel est le seul lézard comestible } \\
\text { lezard rico (les harlicots) } \\
\text { 15. M. Et Mme Célère ont un fils comment s'appelle-t-il? } \\
\text { j'accélère (Jacques Célère) }\end{array}$ \\
\hline Récurrence syllabique (coup) & $\begin{array}{l}\text { 16. Où les supers héros vont-ils faire leurs courses? } \\
\text { Au Super Marché ! } \\
\text { 17. Quelle est la femelle du hamster? } \\
\text { Hamsterdame (Amsterdam) } \\
\text { 18. C'est une feuille de papier qui avance dans l'eau. } \\
\text { Et qui dit au secours j'ai pas pied! (Papier). } \\
\text { 19. Ce matin, j'ai voulu faire une blague sur le Super U, } \\
\text { mais elle a pas Supermarché } \\
\text { 20. Quel super héros donne le plus vite l'heure? } \\
\text { Speed heure man! (spider man ) } \\
\text { 21. C'est un bol et une tasse qui sont devant un évier : } \\
\text { Je n'ai pas envie de plonger, dit le bol, à chaque fois je bois la tasse. } \\
\text { C'est vrai, répond la tasse, tu n'as jamais eu de bol]! }\end{array}$ \\
\hline
\end{tabular}

Comme on a pu le constater, la procédure du jeu syllabique par répétition est moins fréquente dans la création des mot d'esprit que la procédure de dé-contexualisation simple que nous présentons dans la suite.

\subsection{La procédure anaphorique dans l'énonciation des mots d'esprit.}

Les catégories du tableau 2 ont été établies par déviance anaphorique, la réponse à la question est une reprise non autorisée par des éléments linguistiques d'objets disparates dans un même contexte d'énonciation. Ces mots d'esprit sont particulièrement réussis, parce qu'ils engagent la compétence sémiologique à construire des modèles énonciatifs selon les différents contextes.

Tableau 2

Rapports anaphoriques ${ }^{7}$

\begin{tabular}{|c|c|}
\hline MODALITÉ & EXEMPLE \\
\hline $\begin{array}{l}\text { METASTASE } \\
\text { ANAPHORIQUE } \\
\text { (Espace) }\end{array}$ & $\begin{array}{l}\text { 1. Deux ballons sont en train de rire, puis la maman ballon } \\
\text { arrive et leur demande ce qu'il font: } \\
\text { On s'éclate! } \\
\text { 2. Deux oufs se rencontrent : } \\
\text { - T'as l'air brouillé...., } \\
\text { - Je suis crevé, totalement à plat! } \\
\text { 3. Quel est le comble pour un professeur de musique. } \\
\text { Mettre des mauvaises notes. }\end{array}$ \\
\hline
\end{tabular}




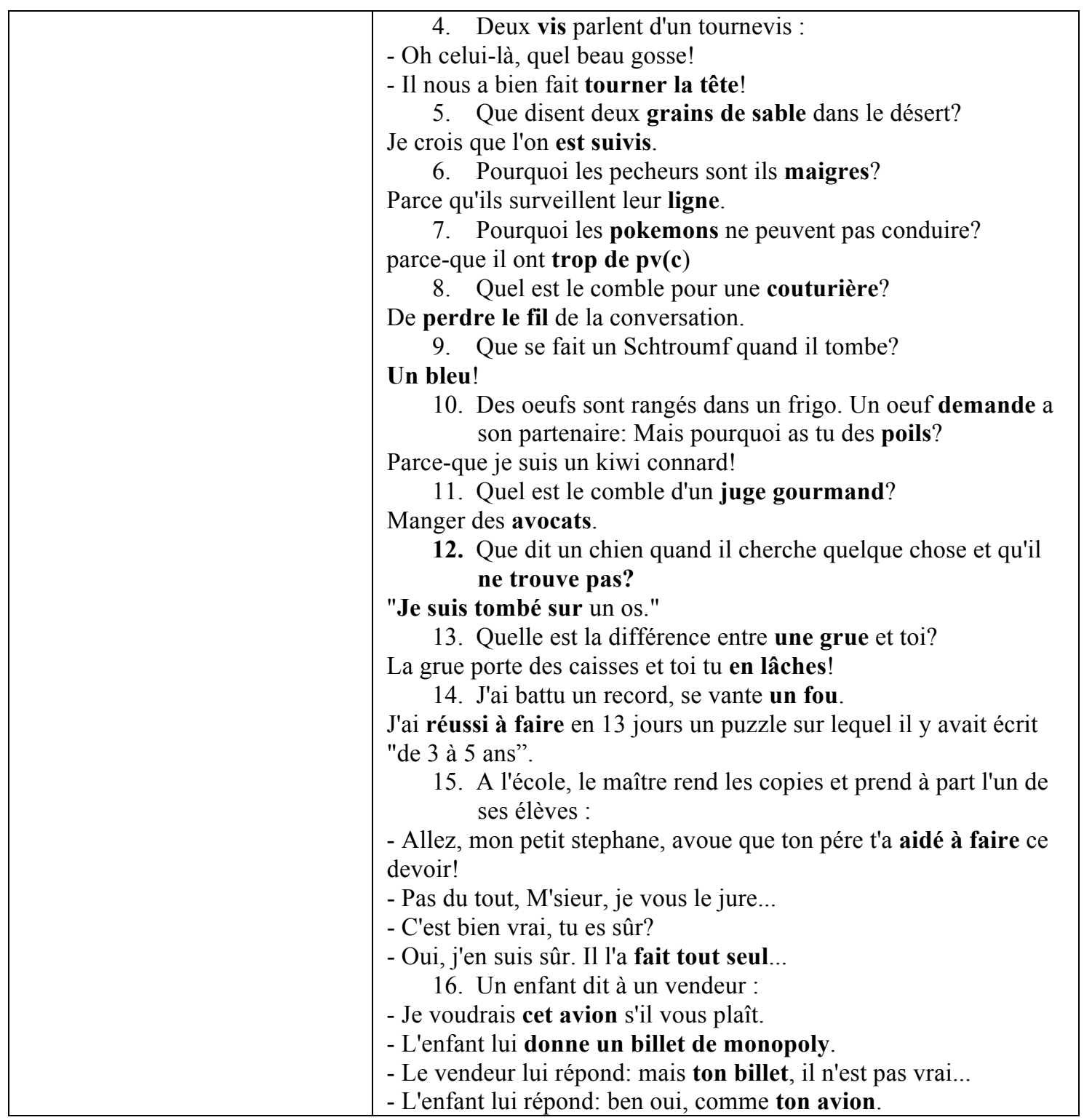

Les exemples ci-dessus montrent une énonciation non habituelle les éléments en caractères gras: (1) les ballons sont des sujets passifs et ne peuvent pas s'éclater dans le sens pronominal; (2) les oeufs n'ont pas l'air brouillés, ils le sont, ils ne se fatiguent pas (ils ne sont pas crevés) et sont sur le plat et non pas à plat. Si on continue à analyser toutes les occurrences en caractères gras, on verra une incohérence d'emploi prédicatif des instances successives. Il est donc raisonnable de penser que la prédication met en rapport des éléments qui le sont déjà dans leur contexte extra-linguistique (Némo: 2003), ce qui nous autorise à dire que l'acte prédicatif met en évidence un rapport sémantique déjà constaté ou, pour le dire autrement, les mots isolés ne signifient que dans le contexte linguistique. Le fait qu'il ait deux grandes catégories bien distinctes dans la structure des jeux de mots (rapports syllabiques et anaphoriques) indique bien que l'une est dépendante de l'autre dans la l'élaboration du discours. Mais alors que les premières sont facilement identifiables par le code phonologique, les deuxièmes (tableau 2) sont difficilement reproductibles et repérables par un système de traduction automatique. C'est le changement 
de point de vue énonciatif qui semble difficile à reproduire. Or, celui-ci est bien présent, comme on va le montrer, dans les verbes par la flexion, ce qui nous permet d'en détecter les locus dans le syntagme.

\section{L'émergence des catégories lexicales à flexion: temps instancié et métastase dans la mémoire phonologique.}

\subsection{La dépendance relative de la métastase des éléments morphologiques}

Ce qui caractérise les trois expressions parémiques ${ }^{8}$ que nous présentons comme modèle de ce qui a été largement travaillé ailleurs, c'est leur capacité à déclencher ce que Nyckees (2000) appelle l'intercompréhension métaphorique. Elles se sont lexicalisées par la récurrence des facteurs extralinguistiques qui les active. Ces énoncés sont alors super-ordonnés (Kleiber: 2005) et restent engrangés dans la mémoire de l'interlangue par la déviance de leur contexte pragmatique d'utilisation. Suivant à nouveau Némo (2003), il y aurait, 3 types de sens dont deux sont conventionnels, les significations linguistiques codées d'une part, les interprétations contextuelles mémorisées d'autre part et les interprétations contextuelles non mémorisés.

Nous considérons des significations linguistiques codées, celles qui sont décrites par leur typologie syllabique tels que les jeux de mots syllabiques analysés en 1.1 et certaines parémies qui mettent en évidence leur caractère formel (redondances lexicales, assonances). En voici quelques exemples de ces dernières ${ }^{9}$ :

(1) C'est bonnet blanc et blanc bonnet

(2) Bon pain se prouve le lendemain

(3) Tel père tel fils

Quant aux interprétations contextuelles mémorisées décrites par la morphologie, on pourrait argumenter leur codification (elles contiennent des régularités syllabiques) mais elles sont surtout des catégories grammaticales construites par le métalangage et ont une extension strictement domaniale, puisqu'elles peuvent être employées en différents contextes par la récurrence des situations qui les déclenchent. Si nous comparons les exemples 4 à 18 aux mots d'esprit recueillis dans le tableau 2, nous pouvons remarquer que les régularités syllabiques affectent des mots susceptibles de présenter une dérivation morphologique qu'ils actualisent différemment dans chaque expression:

(4) Quand les gros maigrissent les maigres meurent

(5) Qui a bu, boira

(6) Les eaux des rivières dorment, l'ennemi ne dort jamais.

(7) Tel est pris qui voulait prendre

(8) Ce qui est pris n'est plus à prendre

(9) Aimer est un plaisir, être aimé est le bonheur

(10) Je n'aimerai jamais, j'aimerai toujours: promise aussi folle l'une que l'autre

(11) Rira bien qui rira le dernier

(12) Le jeune coq chante comme il entend chanter son père

(13) Qui s'écoute parler, n'écoute qu'un sot homme

(14) Le bon parleur a le don de parler pour ne rien dire

(15) Tels parlent sans penser, tels pensent sans parler

(16) Interroge celui qui a éprouvé la maladie, n’interroge pas le médecin

(17) Qui veut mourir ou vaincre, est vaincu rarement

(18) Le chevrotin peut oublier le collier mais le collier n'oublie pas le chevrotin

Nous appelons métastase métaphorique celle qui est évidenciée par la morphologie et répond à notre capacité à construire des catégories sans lesquelles la langue n'existerait tout simplement pas. Les rapports lexicaux se basent sur une linéarité temporelle, car toute linéarisation implique changement à moyen ou long terme (même imperceptible pour les interprètes) dans le bagage commun de la langue. Les 
métastases établissent des rapports méta-phoriques entre les éléments constitutifs des faits linguistiques qui ont été définis d'hyperonymiques, d'hyponymiques ou d'antonymiques. Il est alors possible de les étiqueter pour repertorier des collocations et les catégoriser en termes universels de dépendances lexicales (lexies). Les signifiants émergent alors par leur syntaxe combinatoire, restrictive, sélective ou solidaire (Martin 1979) au niveau de la suite syllabique maximale (une dizaine de syllabes suivant les langues). Dans le cas des phraséologies verbales objet de notre étude, ces critères de sélection restent les mêmes, comme nous allons le montrer en relevant la valeur aspectuelle de leur lexies. Cette valeur peut constituer des entrées de dictionnaires à l'instar de celles que propose Seong Heon Lee (2011: 219-220):

\begin{abstract}
Il a été montré que de nombreux verbes fonctionnent comme verbes supports [Vsup] et que leur fonction sémantique varie selon les nombres prédicatifs avec lesquels ils se combinent pour les "actualiser" ou conjuguer. (...) Vsup estandard (base). Vsup stylistiques (ou variantes lexicales), Vsup aspectuels, Vsup intensifs, Vsup de conversión, Vsup causatifs, Vsup événementiels, Vsup honorifiques...
\end{abstract}

Cette complexité nous révèle les catégorisations possibles des phraséologies à base verbale, mais seulement une fois qu'elles ont été inventoriées. Nous retiendrons dans ce qui suit 2 grandes classifications pour leur inventaire, basées sur l'aspect verbal en spécifiant le trait résultatif (+/- TELEO) pour fixer l'espace énonciatif des signifiés émergents. Nous nous appuyons sur les schémas sémanticocognitifs (SSC en avant) de Desclés (2011) à qui nous avons de même emprunté la notion de télicité. Dans le tableau 3 nous présentons les SSC dans la première colonne que nous associons aux instances de la sémiologie des pratiques ${ }^{10}$ langagières de Coursil (2000) de la deuxième colonne. La troisième colonne spécifie les rapports sémantiques qui se produisent dans les lexies qui peuvent être combinés entre eux pour former des énoncés à longueur variées, des textes ou des discours selon le type des périodes énonciatives que nous analysons. Nous ne pouvons ici avancer dans les catégorisation sémantique des exemples de phraséologies verbales qui reste un long travail a entreprendre après la constitution des répertoires. Le cadre théorique pour l'élaboration des répertoires est présenté dans le tableau ci-dessous où les coïncidences entre SSC et la sémiologie s'explicitent par leur dépendance dans la linéarité du signe linguistique qui produit des énoncés selon les contraintes syllabiques.

Tableau $3^{11}$

Rapports SSC/ Sémiologie des pratiques et types de périodes énonciatives

\begin{tabular}{|l|l|l|}
\hline SSC & Sémiologie des Pratiques & Périodes énonciatives \\
\hline 1 Relateurs statiques & $\begin{array}{l}\text { Icône visuelle } \\
\text { (ESPACE) }\end{array}$ & $\begin{array}{l}\text { Unité de discours } \Leftrightarrow \\
\text { (Combinaison) }\end{array}$ \\
\hline 2 Relateurs cinématiques & $\begin{array}{l}\text { Effets prosodiques typologiques } \\
\text { de syllabation } \Rightarrow \text { (sélection) }\end{array}$ \\
\hline $\begin{array}{l}\text { 3 Relateurs dynamiques } \\
\text { [+/-TÉLÉO] }\end{array}$ & $\begin{array}{l}\text { Dérivation lexicale } \\
\text { (GESTE) }\end{array}$ & $\begin{array}{l}\text { Lexie à morphèmes } \\
\text { (restriction) }\end{array}$ \\
\hline $\begin{array}{l}\text { 4 Relateurs représentationnels } \\
\text { Simples }\end{array}$ & $\begin{array}{l}\text { Polarité par compositionnalité } \\
\text { (CHOC) }\end{array}$ & $\begin{array}{l}\text { Valeur par contraste (polarité) } \\
\text { / } \Rightarrow\end{array}$ \\
\hline 5 Relateurs multiples & $\begin{array}{l}\text { Énonciation endophasique } \\
\text { (ÉTENDUE) }\end{array}$ & $\begin{array}{l}\text { Marqueurs textuels de modalité } \\
\text { récurrents } \Rightarrow \Rightarrow \text { solidarité) }\end{array}$ \\
\hline Énonciation par instances & $\begin{array}{l}\text { Énonciation par gradients/ } \\
\text { dialogisme } \\
\text { (MOUVEMENT) }\end{array}$ & $\begin{array}{l}\text { Agents, Compléments, discours } \\
\text { direct/indirect ... } \Leftarrow \Leftarrow\end{array}$ \\
\hline
\end{tabular}

Comme nous le voyons, lesschémas cognitifs de Déclès, peuvent être interprétés dans un cadre sémiologique de dépendances syntaxiques. Nous allons montrer dans ce qui suit que cette sémiologie est repérable par le caractère morpho-syllabique des énoncés isolés par leur prosodie ${ }^{12}$ dans un espace dialogique. Les exemples 5 à 18 ci-dessus sont désormais analysables et classifiables en sous-catégories 
selon les principes SSC (2, 3 et 4), les relateurs cinématique, dynamiques et représentationnels. Quand aux autres relateurs du tableau 3, ils affectent des unités non mémorisées (Némo: 2003) et correspondent dans la langue au discours "non-répété", pour reprendre la terminologie de la parémiologie. On conclut donc que les relateurs encodant les séquences méta-phorique de ces quelques exemples de notre corpus sont cinématiques (reproduisent des effets syllabiques dans le même segment), dynamiques (ont été encodés comme des formes à dérivation) ou représentent un effet de contraste. Ces caractéristiques vont servir de critère à la catégorisation des phraséologies verbales.

\subsection{Ouverture/fermeture énonciative: éléments prosodiques des faits linguistiques}

L'étude des expressions idiomatiques invite à élargir le cadre de la catégorisation grammaticale pour expliquer les faits de langue. Selon Nyckees (2000: 21) l'ordre catégoriel n'est pas une doxa figée: dans cette perspective, nous allons le voir, la conception de répertoires d'expressions verbales reposent tant sur la notion grammaticale d'aspect que sur des arguments propres à l'analyse du discours métaphorique. Considérons les expressions (4) à (18) du tableau suivant: elles reposent tant sur la morphologie verbale que sur des récurrences syllabiques. Ces deux caractéristiques intrinsèques à ces expressions produisent des clusters intonatifs qui les isole du co-texte. Le système catégorial de la grammaire s'enrichit alors par des topiques du bagage linguistique d'une communauté en assumant les référents culturels sur lesquels reposent des faits linguistiques idéologiques fréquents dans une communauté linguistique. Ainsi les métaphores ne sont pas libres et doivent se construire sur une catégorisation énonciative commune, car:

Apprendre à métamorphiser, c'est peut-être, à certains égards, se couler dans un moule contraignant, apprendre les rigueurs de l'expression, mais aussi et surtout apprendre à penser, s'ouvrir à une infinité de nouvelles connexions, de nouveaux modes de catégorisations de l'expérience [Nyckees, 2000: 137]

Voyons donc maintenant dans le tableau 4, les rapports métaphoriques basés sur des instances temporelles codés par la morphologie verbale des expressions (4) à (18):

Tableau 4:

Dépendances à métastase temporelle

\begin{tabular}{|c|c|c|}
\hline $\begin{array}{l}\text { CATÉGORISATION } \\
\text { MORPHO-LEXICAL }\end{array}$ & $\begin{array}{r}\text { EX./ } \\
\mathbf{N}^{\circ}\end{array}$ & $\begin{array}{lr}\text { RAPPORT: } \quad \text { R/réciproques: } & \text { rapport } \\
\text { hyperonymique-/Hyponymique } & - \\
\text { A/antonymique } & \\
\end{array}$ \\
\hline Passé/Futur & 5 & $\mathrm{R}$ (Buveurs: humains) $\Leftarrow$ \\
\hline Vérités générales + polarité & $4-6$ & A (Contraires) $/ \Rightarrow$ \\
\hline Passive présent/active modale au passé & 7 & R (Réciproques - double prédication) \\
\hline Vérité générale/Passive de constat + polarité & 8 & A (Contraires) $/ \Rightarrow$ \\
\hline Active vérité générale/Passive vérité générale & 9 & R (Aimants, aimés: humains) \\
\hline Futur d'intention+polarité & 10 & A (Contraires) $/ \Rightarrow$ \\
\hline Futur de projection & 11 & R (Humains: rieurs) \\
\hline Rapport présent/verbe modal & 12 & $\mathrm{R}$ (Coq: poussins) \\
\hline Vérité générale réfléchie & 13 & R (Humain: individu) \\
\hline Changement de statut lexical + polarité & 14 & A (Contraires) $/ \Rightarrow$ \\
\hline Rapport présent/infinitif & 15 & R (Réciproques - double prédication) \\
\hline Impératif + polarité & 16 & A (Contraires) $/ \Rightarrow$ \\
\hline Rapport verbe modal/passif & 17 & R (Réciproques - double prédication) \\
\hline Rapport verbe modal/présent+polarité & 18 & R (Réciproques - double cible énonciative) \\
\hline
\end{tabular}

On voit que la morphologie du verbe dans la lexies établit des catégories domaniales qui correspondent aux intuitions du locuteur par la seule référence au cadre (morphologique) imposé par une grammaire collective. Paradoxalement, le cadre présenté rend possible la transposition à toutes les langues de leurs 
catégorisations car son caractère reste sémiologique pour les effets produits par les redondances syllabiques (exemples 1 à 3), morphologiques des exemples suivants ou métastasiques du tableau 1. Elles sont mémorisées par la référence à une confrontation de deux éléments à morphologie. C'est donc bien la mémoire phonologique qui agit dans la production du message, ou pour le dire autrement, la syllabation reproduit des constantes phonologiques emmagasinées dans un langage commun fait de faits linguistiques catégorisés dans le cadre, linguistique lui aussi, du métalinguistique. Les constantes phonologiques concernant la morphologie sont interprétées dans un espace énonciatif borné et sont alors conclusives et redondantes par rapport au contexte qui les explicite. Cela implique une ouverture énonciative associée à un contexte discursif et une fermeture conclusive que la prosodie marque d'un intonème final descendant ${ }^{14}$.

Nous appliquons, dans ce qui suit, ces critères de catégorisation à des expressions verbales. Pour cela on fait intervenir une notion d'aspect élargie.

\section{Les idiomes à base verbale et le trait +/- télicité dans les collocations: les exemples de llevar, dar, tomar, coger, poner, soler.}

Nous traitons dans ce qui suit les phraséologies verbales comme des clusters construits dans la linéarité du procès de syllabation en tenant compte d'un point de référence (instantiation), d'un moment événementiel et de l'énonciation en elle même ${ }^{15}$. Leur caractère sémiologique est donc repérable dans la linéarité de la chaîne verbale, ce qui les rend arbitraires comme le signe saussurien qui présente une étendue, (...) mesurable dans une seule dimension ${ }^{16}$.

Dans cette perspective d'extension linéaire, l'intervention active du sujet énonciateur actualise/interprète un cadre sémiologique ancré dans la morphologie. Il s'agit de l'émergence de ce que Némo appelle interprétations contextuelles non mémorisées (Voir ci-dessus). Ce sont des rapports métaphoriques qui sont à mi-chemin entre le figement et le défigement, et constituent le domaine des collocations/colligations verbales. Ces expressions présentent une ouverture sur l'allocutaire qui devra reconnaître au moment de l'énonciation la pertinence de l'information. Pour cela, l'aspect de télicité doit être confirmé par le contexte extra-linguistique.

Une simple analyse contrastive de « avoir raison » [llevar razón / tener razón] et « avoir tort » [no llevar razón/no tener razón] indique la dépendance de ces expressions de la situation d'énonciation. Considérons les exemples suivants :

(19) Tiene razón $\rightarrow$ Il a raison (perfectif $\rightarrow$ perfectif)

(20) No tiene razón $\rightarrow$ Il n'a pas raison [emphase] (perfectif $\rightarrow$ perfectif)

(22) Lleva razón $\rightarrow$ Il n'a pas tort (imperfectif $\rightarrow$ perfectif)

(23) No lleva razón $\rightarrow$ Il a tort [emphase] (perfectif $\rightarrow$ perfectif)

Comme on le voit en (22) le verbe « llevar» suggère un sens imperfectif para contraposition à "Tener » [avoir] de (1). Mais la traduction française ne peut pas rendre cela car la négation entraîne marquer d'un trait + accompli l'énoncé (ne pas avoir tort). En (20) en effet, la négation du verbe entraîne une emphase qui s'opposerait à tout argument postérieur, comme c'est le cas de 23 (trait perfectif).

Les idiomes et énoncés figés en général dévoilent l'intention chez l'utilisateur de marquer son argument d'un point final. En cela la notion de télicité aide à comprendre leur structure bornée. Nous voyons un exemple dans l'œuvre la plus parémique répertoriée dans l'histoire de la langue espagnole, Le Quichotte ; pour détourner son maître de la folie, Sancho Panza utilise souvent des parémies; ces idiomes même enchaînés entre eux ont le but de ne pas laisser passer les arguments de son maître aliéné dans les lectures ${ }^{17}$. Par métastase temporelle, c'est-à-dire par désancrage temporel par rapport à la situation d'utilisation, certaines formes verbales acquièrent des valeurs grammaticales spécifiques interprétables par une notion d'aspect élargie, celle de télicité. Ainsi, l'auxiliaire « llevar » implique une indécision du locuteur qui n'ose pas affirmer de façon abolue. C'est cet aspect imparfait de l'affirmation (non télique) qui conduit à la pertinence de la traduction de « llevar razón » par « ne pas avoir tort » et non par « avoir raison » dont le trait de télicité (affirmation sans réplique possible) ne correspond pas au sens de «llevar razón » qui s'ouvre à un horizon d'explications. (19), (20) et (21) sont des énoncés assertifs marqués de 
télicité positive pour differentes raisons : 19 et 20 pour la spécificité de l'idiome, (21) pour la négation car elle découpe un fait de langue dans la suite énonciative comme l'ont montré les études sur les adverbes duratifs (Culioli:1999; Renaud:2005)

On peut dire que la lexie dont fait partie l'auxiliare l'emporte sur l'opposition affirmatif/négatif et doit rester alors un trait d'interprétation contextuelle et non un fait de langue codifié ou mémorisé avec son contexte comme c'est le cas des idiomes ou parémies.

Dans cet ordre de choses, comme on va le voir, l'emploi du pronom réfléchi «se » est un autre fait intéressant à remarquer dans les phraséologies verbales (voir 3.2): tout comme la négation, il a une fonction polaire non seulement dans le cas des parémies (voir exemple 13) mais aussi dans l'interprétation des phraséologies verbales (collocations en avant).

Les cas de collocations avec « llevar», « dar», " tomar», « coger», « poner » et " soler» semblent partager des caractéristiques avec les énoncés que l'on a présentés ci dessus. De même, l' 'impossibilité de disposer de traductions basées sur un même auxiliaire en français, indique bien que la collocation n'a pas de base lexicale, et que ce ne sont donc pas à proprement parler des « figements » mais des colligations : elles ont émergé dans la langue, non seulement pour leur typologie syllabique mais aussi par les catégorisations grammaticales qui ont organisé la morphologie. Malgré leur apparence d'expressions faites, ces structures verbales exigent un mouvement actif de type énonciatif, un geste conclusif ou non conclusif que nous marquons par \# $\left[\Leftarrow{ }^{*}+\right.$ Téleo $\left.^{\mathrm{x}}\right], \#\left[\Leftarrow{ }^{*} \text {-Téleo }{ }^{\mathrm{x}}\right]^{18}$.

Reprenons quelques exemples d'expressions ou l'aspect télique est présent :

\subsection{Exemples marqués de télicité négative :\# $\left[\leftarrow^{*}-\right.$ Téleo $\left.^{x}\right]$}

Llevar:

(24) Llevar una tajada [Etre ivre-mort]

(25) Llevar la voz cantante [Commander/ne pas se laisser faire]

(26) Llevar adelante [Faire avancer]

Dar:

(27) Dar la lata [casser les pieds]

(28) Dar razón de algo [renseigner]

(29) Dar la impressión* [sembler]

Tomar:

(30) Tomar el sol [prendre le soleil]

(31) Tomar/coger al toro por los cuernos [affronter une situation]

(32) Tomar algo [boire un coup]

Coger:

(33) Coger un resfriado [s'enrhumer]

(34) Coger el guante [accepter le défi]

(35) Coger al rábano por las hojas [faire très attention]

Poner:

(36) Poner a caldo [insulter grièvement]

(37) Poner la mano en el fuego [montrer sa confiance pour]

(38) Poner la zancadilla [mettre des bâtons dans les roues]

\subsection{Exemples de télicité positive : \#[ $\leftarrow^{*}+$ Téleo $\left.^{x}\right]$}

Llevarse:

(39) Llevarse un susto [s'effrayer]

(40) Llevarse una tajada [Avoir une tranche]

(41) Llevarse el gato al agua [L'emporter sur quelqu'un]

Darse:

(42) Darse por vencido [se déclarer vaincu]

(43) Darse por enterado [montrer qu'on a été informé] 


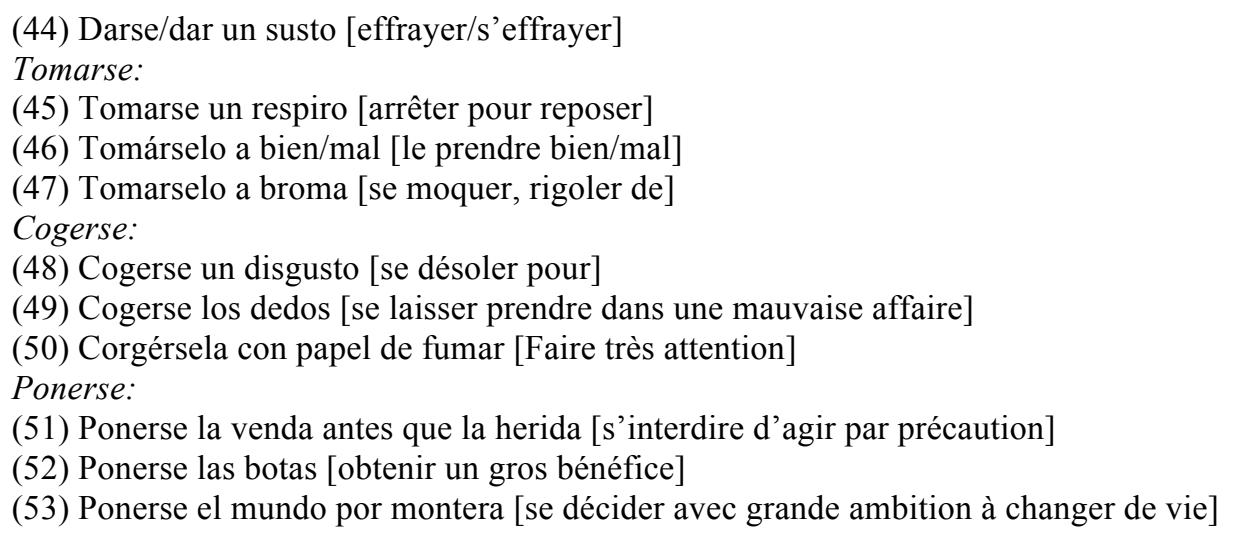

À différence des formes proverbiales (contenant ou non des éléments morphologiques) qui peuvent être repérées par leur détachement du reste du discours et leur autonomie relative, les expressions à base morphologique gardent un caractère télique particulier. Elles dépendent d'un verbe ou d'un adjectif (muerta de risa [morte à force de rire] /rouge comme un coquelicot) qui leur confère un locus prosodique particulier (intonème conclusif), ce qui les différencie d'autres éléments à morphologie faisant partie de lexies dans différents contextes. Cet aspect prosodique ne leur permet pas changer de statut dans le discours, comme c'est le cas dans les lexies isolées, qui peuvent être sujet de recatégorisation grammaticale par leur position instable dans l'énoncé. Nous argumentons que le trait de télicité sert à construire des unités lingüistiques dont le caractère est à mi-chemin entre le mot lexical et les expressions métaphoriques. Ces unités sont détectées par le locuteur natif pour leur caractère prosodique (trait final conclusif) et leur nature doit être explicitée en termes de grammaire. Un grand travail sur corpus reste néanmoins à faire pour justifier leur caractère prosodique indépendant du reste du discours.

\subsection{Les exceptions qui confirment la règle :}

Un autre argument qui nous semble valider notre conception des phraséologies verbales comme dépendant d'un contexte domanial (Kleiber) répondant à l'aspect télique et susceptibles d'être repérées par leur trait intonatif conclusif, est le fait qu'il existe des expressions à sens passif du type poner + adjectif [rendre+adjectif] et dar+nom [faire+nom] qui ont déjà été grammaticalisées et qui disposent de correspondances en français/espagnol marquées par le même type de télicité. Ces phrasèmes, dits causatifs, sont en réalité des lexies à type résultatif:'

(54) poner enfermo/nervioso [rendre malade/nerveux]

(55) Dar miedo/ganas [faire peur/envie]

Considérons encore ces exemples avec "soler" [avoir l'habitude de]; ils sont typologiquement marqués d'un trait de télicité négative \# [ $\leftarrow^{*}$ - Téleo ${ }^{\mathrm{x}}$ ], puisqu'ils réfèrent les faits habituels. Les traductions sont encore une fois diverses car elles concernent des catégories où l'aspect duratif (duracité dans la terminologie de Renaud) est présent:

(56) Solíamos ir en metro [Nous avions l'habitude d'aller en métro].

(57) No solía comer mucho [Il ne mangeait pas beaucoup]

(58) Suele llegar sobre las 10 [Il vient normalement vers $10 \mathrm{~h}$ )

On voit, par les traductions, que l'aspect de duracité de cette expression est riche et que les inflexions morphologiques reproduisent les constantes temporelles dans des instances bornées par un terme nucléaire dans la lexie. En effet, selon Vendler (1957) ${ }^{19}$ on peut fonder des classifications sur la base de la durée (duracité) sur l'opposition des types de procès (...) procès dynamiques bornés, et (...) activités et états $^{20}$ 
Le corolaire à notre travail est que les procès dynamiques bornés donnent lieu à de collocations dans le sens large du terme. Or, établir des bornes dans le processus d'énonciation semble bien propre de toute activité linguistique étant probablement à la base de l'activité prédicative. En effet, articuler le débit syllabique en périodes plus ou moins longues et dépendantes de constantes physiques (respiration, procédures de mise en mémoire) c'est peut-être construire des phrases dans le texte ou des énoncés dans le discours, c'est à dire qu'une:

Une phrase est définie par les règles de bonne formation qui régissent essentiellement une relation prédicative; un énoncé est une relation prédicative repérée par rapport à un système de coordonnées énonciatives. [Culioli, 1999:129]

Autrement dit, il faut un localisateur qui se déplace dans un schéma lexical qui n'est qu'un segment linéaire de variables ${ }^{21}$.

Ce schéma convient à notre proposition de classification de paraphrases verbales, car la notion de télicité peut bien entrer dans les procès d'achèvement et d'accomplissement (télicité positive et négative respectivement) propre des expressions périphrastiques. Cela nous permet de considérer des segments linguistiques commençant par un élément morphologique et clôturé par une inflexion prosodique, qui par convention, nous symbolisons par \#, fin de l'énoncé. À l'intérieur peuvent jouer de représentations définissables tout probablement en termes de solidarités sémantiques telles que celles que nous avons décrites (tableau 4) et qui émergent des rapports temporels des éléments segmentaux dans un cadre sémiologique large (tableau 3).

\section{Conclusions}

Le métalangage classique de la grammaire qui permet la classification d'élements lexicaux ne semble pas pouvoir expliquer la variété des lexies engrangées dans la langue et en constante formation et reformulation. Les groupements syntaxiques qui dépendent de la volonté énonciatrice d'un sujet dans un instant $\mathrm{T}_{0}$ et une situation $\mathrm{S}_{0}($ Culioli, 1999:129) dépendent:

1. D’un cadre sémiologie primitif.

2. De l'espace énonciatif ou période conclusive (+/- Téleo).

3. Des péculiarités syllabiques arbitrairement motivées dans la linéarité de la diachronie d'une langue.

Nous défendons la pertinence de la notion de période énonciative qui délimite un espace sémiologique borné dont nous avons présenté ici et ailleurs quelques exemples par des idiomes présents dans toutes les langues (jeux de mots, parémies, phraséologies verbales). Notre hypothèse est que la mémoire phonologique, limitée par des constantes physiologiques (respiration, limitations phono-tactiques) produit des segments catégorisables dans et par leur espace intono-accentuel. Cet espace intono-accentuel ou période énonciative n'est abordable qu'à travers les faits linguistiques qu'il génère et les catégorisations grammaticales avec lesquelles nous les répertorions. Or un fait linguistique n'est pas un fait de langue figé. Le figement n'est pas propre au langage humain. La compétence topique dont parle Nyckees (2000), trace les limites de la traductibilité mais garantit de même le capital symbolique commun où toutes les langues baignent et dont le témoignage plus évident sont ces dé/figements expressifs. Nous avons abordé ces instances par leur caractère syntaxique selon une articulation de dépendances et solidarités propre à tous système syllabique. Le résultat devrait permettre de transposer les procédures de classification de langue à langue (métastase métaphorique) et d'avancer dans l'implémentation de systèmes de traduction virtuels, suivant les pistes de Némo (2003: 93), par la description de la signification linguistique (analyse contrastive des faits de langue), l'interprétation contextuelle (définition de l'espace énonciatif où ils se produisent) et la description du processus de mémorisation des interprétations contextuelles, autrement 
dit, des processus de lexicalisation (catégorisation des segments syllabiques par délimitation des périodes prosodiques).

\section{Références bibliographiques}

Abreu, R.; Escobar, M. C. A. (2009). Sistema de verificación de locutor basado en modelos ocultos de Markov, Universidad Experimental Nacional Politécnica Antonio José de Sucre. http://www.el.bqto.unexpo.edu.ve/rabreu/Investigacion/TEG1.pdf

Avanzi, M.; Lacheret-Dujour, A. ; Obin, N. ; Victorri, B. (2011). Vers une modelisation continue de la structure prosodique: le cas des proeminences syllabiques. French Language Studies, n. ${ }^{\circ}$ 21, 53-71.

Barsanti Vigo, M.J. (2006) Cinco refranes retóricos de Sancho en El Quijote de Ludwig Tieck. Paremia, 15: 2006, p179-186.

Corpas Pastor, G. (1997). Manual de fraseología española. Madrid: Gredos.

Coursil, J. (2000). La fonction muette du langage. Guyane : Ibys rouge.

Crida Alvarez, C.A. \& SEVILLA, J. (2013). Las paremias y su clasificación. In Paremia, 22: 2013, pp. 105-114.

Culioli, A. (1999). Pour une linguistique de l'énonciation. Paris, Ophrys.

Desclés, J.-P. (2011). Le problème de la polysémie verbale : DONNER français. In Seong Heon Lee (Dir.). In Seong Heon Lee (Dir.) Du lexique au dictionnaire en passant par la grammaire. Cahiers de Lexicologie, 95-1 : 2011, pp. 95-111.

Encrevé, P. (1988). La liaison avec et sans enchaînement. Phonologie tridimensionnelle et usages du français. Paris: Seuil.

Fernández, M. L. (2014). Clasificación de unidades fraseológicas francesas según su peculiaridad silábica. Paremia, n. ${ }^{\circ} 23,91-99$.

Fernandez, M. L. (2015b). La lutte des classes des unités linguistiques: micro et macro-syntaxe. In Studii de lingüística, 5/2015, 179-202.

Fernandez, M. L. (2016a). L'aspect : ser/estar en espagnol. L'aspect dans les langues naturelles: comparaisons langues romanes/autres langues, Studii de stiinta si Cultura (sous presse).

Fernández, M. L. (2016b). Criterios para el estudio contrastivo de unidades lingüísticas españolas y francesas a caballo entre coligación y colocación. Paremia (Sous presse).

García Yelo, M. (2011). El proceso de desautomatización de las paremias españolas en las redes sociales. M. ${ }^{a}$ Isabel González Rey (ed.). Unidades fraseológicas y Tic. Madrid: Instituto Cervantes, 111-124.

Gardes Tamin, J. (2011): La grammaire. Paris. Armand colin.

Herslund, M, (2012). A quoi bon le mot? Réflexions sur sémantique et lexicologie, in Begoni \& Bracquener (dir.) Sémantique et léxicologie des langues d'Europe, théories méthodes et applications. Rennes: PUR.

Kleiber, G. (2015). La figure du proverbe n'est pas toujours celle d'une métaphore. Communication orale: Sémantique, syntaxe et pragmatique du proverbe. Université Complutense de Madrid, 8-9 octobre 2015. 
Kleiber, G. (1999), La sémantique du prototype, Paris: PUF.

Laks, B.(1997), Phonologie accentuelle : métrique, autosegmentalité et constituance, Paris, CNRS.

Legallois, D. (2012). La colligation : autre nom de la collocation grammaticale ou autre logique de la relation mutuelle entre syntaxe et sémantique?», Corpus, n. ${ }^{\circ} 11,31-54$. En ligne : http://www.crisco.unicaen.fr/IMG/pdf/La colligation autre nom de la collocation grammaticale.pdf

Martin, Ph. (1979). Une théorie syntaxique de l'accentuation en Français. In I. Fonagy y P. Léon (dirs.) L'accent en Français contemporain. Otawa: Marcel Didier, 1-12.

Mejri, S. (2011). Collocations et emplois appropriés : des unités lexicales hybrides?, in Seong Heon Lee (dir.) $D u$ lexique au dictionnaire en passant par la grammaire. Cahiers de Lexicologie, 95-1 : 2011, pp. 84-94.

Némo, F. (2003). Indexicalité, unification contextuelle et constitution extrinsèque du référent. In Langages, nº17.

Nyckees,V. (2000). Quelle est la langue des métaphores? In Cahiers de praxémique: Sens figuré et figuration du monde, 35, pp. 114-139.

Penadés Martínez, I. (2012). La fraseología y su objeto de estudio. Linred, 10, en ligne : http://www.linred.es/monograficos pdf/LR monografico10-articulo2.pdf

Renaud, R. (2005). Temps, durativité, télicité. Louvain : Peeters - Paris - Duddley.

Saussure, F. (1915). Cours de linguistique générale. Paris: Éditions Payot, 1972-1985.

Seong Heon Lee (2011). Le dictionnaire électronique Sejong revisité. in Seong Heon Lee (Dir.) Du lexique au dictionnaire en passant par la grammaire, Cahiers de Lexicologie, 95-1 : 2011, pp. 213-231

\footnotetext{
${ }^{1}$ Fernández (2014)

${ }^{2}$ Pour des explications éclairantes sur ce sujet nous référons aux études de la constituance syllabique de Laks (1997) développés par l'équipe PFC http://www.projet-pfc.net/.

${ }^{3}$ Voir Encrevé (1988).

${ }^{4}$ Nous avons consciemment choisi un corpus de blagues d'enfants pour éviter de tomber sur des expressions artificielles, volontairement formulées sur des algorythmes variés comme ceux utilisés en publicité ou en marketing. Nous avons dû évidemment corriger les erreurs orthographiques avant de réécrire sur notre travail ces quelques exemples consultables en ligne: http://www.blablagues.net/blagues-carambar.html

${ }^{5}$ Transposition de constantes phonologiques d'un contexte domanial à autre par un processus de mémorisation de rapports morphologiques opérant dans les lexies.

${ }^{6}$ Les mots d'esprit en cursive ne sont pas du corpus "carambar".

${ }^{7}$ En analysant le type de blagues de notre corpus complémentaire, on retient que les non-syllabiques sont de loin les plus fréquentes: du total du corpus (82 occurrences), seulement $19(21.1 \%)$ répondent à des critères syllabiques strictes. Il est cependant évident que les critères peuvent se superposer [16].

${ }^{8}$ Expressions parémiques: sous-classe des expressions proverbiales à deux termes. Voir Crida Alvarez \& Sevilla (2013).

${ }^{9}$ Nous présentons seulement 3 exemples pour justifier les catégories qui sous-tendent l'analyse qui suit. Le caractère formel de ces parémies a été explicité dans le détail ailleurs (Fernández 2014/2015a, b, c).

${ }^{10}$ Expression empruntée à Coursil (2000)

Unión de segmentos silábicos con valor iconográfico que corresponden a grupos de entonación simples de 7 a 8 sílabas en todas las lenguas según Martin (2009). Estos segmentos se pueden modular y combinar para constituir frases complejas, textos o discursos, siempre con los mismos principios.

${ }^{11} \mathrm{Ce}$ tableau a été conçu dans un autre premier travail sur les phraséologies verbales à paraitre chez Parémia (Fernandez, 2015c)
} 
${ }^{12}$ Ils sont en effet repérables par un effet intono-accentuel qui les détache du reste de l'instance discursive: un accent initial et un trait conclusif les délimite, ce qui les signale comme expressions toutes faites.

${ }^{13}$ Voir: Gardes Tamine (2011:134-135). Nous considérons polaires toutes les relations antonymiques et retenons seulement ces deux catégories (antonymiques et réciproques) pour économiser les rapports métastasiques de notre approche.

${ }^{14}$ Le présent travail ne peut inclure, faute de place, des justifications de ce fait, ce n'est qu'une suggestion d'ébauche de future recherches. Il est cependant évident que les répertoires de périodes énonciatives doivent être bien établis avant de l'entreprendre, ce à quoi nous nous impliquons dans ce travail.

${ }^{15}$ Calendérique dans la terminologie de Renaud, 2005 qui cite par ailleurs Reichenbach:1947)

${ }^{16}$ Saussure (1915).

${ }^{17}$ Y con esto Dios te dé salud y a mi no olvide. Vale. [Proposition personnelle de traduction: Et sur cela, le bon Dieu te donne grâce et n'oublie pas ces autres ouailles. Adieu]; fin de la préface du Don Quichotte. Voir aussi http://www.paremia.org/wp-content/uploads/18-Barsanti.pdf

${ }^{18}$ La diérèse indique le début d'une expression phrastique, la flèche la direction du figement (l'auxiliaire commande); l'astérisque anonce le trait positif ou négatif de l'aspect conclusif et l'exposant le nombre de positions syllabiques jusqu'à la fin du geste énonciatif dans l'expression réelle. Voir Fernández (2015b).

Les phraséologies avec llevar sont encore difficiles à trouver, car il n'existe pas encore, à notre connaissance, des répertoires sur les expressions que nous présentons, ce qui fait l'intérêt du travail. Pour des exemples voir les liens de Linguee et le suivant très expressif:

- http://www.linguee.es/espanol-ingles/traduccion/llevar+la+raz\%C3\%B3n.html

- http://www.jotdown.es/2014/02/el-dificil-arte-de-llevar-razon-estando-totalmente-equivocado/

19 Cité par Renaud, [2005: 253] ; Vendler (1957), "Verbs an Times, The Philosophical Review.

${ }^{20}$ Formulées par Renaud, selon et d'une façon plus linguistique par Dowty en 1979. Ainsi se dégagent 4 catégories: ACH. [Achèvement: Exploser], ACC. [Accomplissements: aller au bureau], ACT. [Activité : Nager], ETAT [États : malade]

${ }^{21}$ Ce qui a été défini par l'auteur comme: $\lambda \underline{\varepsilon} \operatorname{SIT}_{0}\left(\mathrm{~S}_{0}, \mathrm{~T}_{0}\right)$, ou le "lekton $\lambda$ " (lexie) joue un rôle pragmatique et obient un score. Le segment linéaire $\left\langle\xi_{0} \xi_{1} \pi>\right.$ est un segment de variables argumentatives ou $\pi$ est la prédication. Cette prédication est ouverte dans les expressions non mémorisées et fermée dans les expressions mémorisées, codées ou morphologiques. 